also reported bitemporal hypometabolism on PET in 18 infants with spasms and severe developmental delay (Ann Neurol 1996;39:643-649).

\title{
DEVELOPMENT OF WEST SYNDROME IN INFANTS WITH EPILEPTIC DISCHARGES
}

Of 45 infants having epileptic discharges on the EEG in early infancy, 26 developed West syndrome (WS), in a study at Okayama University, Japan. Infants with periventricular leukomalacia (PVL) were more likely to develop WS than those without; they were also more likely to have WS than other types of epilepsy. Eleven out of 17 infants with PVL developed WS. Most infants with WS had developed initial epileptic discharges before 3 months of age. Preterm infants with PVL who show epileptic discharges on EEG before 3 months of age should receive antiepileptic treatment to prevent the onset of WS. (Yoshinaga $\mathrm{H}$, Endo F, Kikumoto $\mathrm{K}$ et al. Epilepsy development in infancy with epileptic discharges. Brain Dev May 2007;29:217-223). (Respond: Dr Harumi Yoshinaga, Department of Child Neurology, Okayama University Graduate School of Medicine, Japan).

COMMENT. A new model of flexion spasms involving prenatal exposure to betamethasone followed by injection of N-methyl-D-aspartic acid (NMDA) on day 15 postnatally in rats may provide a means of development and testing of new effective therapies for infantile spasms (Vellsek L et al. Ann Neurol Feb 2007;61:109-119; Baram TZ. Editorial Ann Neurol 61:89-91). These experimental spasms were sensitive to. ACTH therapy, ictal EEG electrodecremental or afterdischarges compatible with hypsarrhythmia were recorded, and imaging studies defined lesions in the limbic areas, hypothalamus, and brainstem associated with spasms.

\section{PROGNOSIS OF PARTIAL EPILEPSY PREDICTED BY MRI AND PET}

The evolution of cerebral glucose metabolism after partial seizure onset was studied in 38 children using PET scans over $3.0+/-1.3$ years (and within a year after a third unprovoked partial seizure) by researchers at the Clinical Epilepsy Section, NINDS, and Children's National Medical Center, Washington, DC. Twenty-eight patients with an initial normal PET were significantly more likely to have good seizure control than the 10 with initial abnormal scan. Those that developed an abnormal PET had longer history of epilepsy before the first PET but not greater seizure frequency. Patients with shorter time between seizures and higher seizure frequency had a greater risk of abnormal PET. Six of 7 patients with persistently abnormal PET had poor seizure control. History of febrile seizures did not affect PET findings. MRI was strongly predictive of initial PET results, but did not correlate with fluctuating hypometabolism. The combination of MRI and initial PET was strongly predictive of clinical course. (Gaillard WD, Weinstein S, Conry J et al. Prognosis of children with partial epilepsy. MRI and serial FDG-PET. Neurology February 2007;68:655-659). (Reprints: Dr William D Gaillard, Children's National Medical Center, 111Michigan Ave, NW, Washington, DC 20010).

COMMENT. Initial, but not serial, MRI and FDG-PET combined may be useful in predicting prognosis of partial epilepsy in children. 